\title{
Short-Range Detection System with Polarized Laser and Polarization Characteristics of Typical Targets
}

\author{
Yuying Yang, Huimin Chen, \\ Hejiao Chen \\ State Key Lab of Mechatronics \\ Engineering and Control, \\ Beijing Institute of Technology \\ Beijing, 100081, China \\ e-mail: dn20040620@163.com
}

\author{
Yuying Yang \\ Department of Arms Engineering, \\ Academy of Armored Force \\ Engineering \\ Beijing, 100072, China \\ e-mail:dn20040620@163.com
}

\author{
Yunfeng Shao \\ Langfang Campus, \\ Nanjing Artillery Academy \\ Langfang, 065000, China \\ e-mail: wonder1982@sina.com
}

\begin{abstract}
In view of using only the intensity information of retroreflection light and poor capacity of anti-interference in laser short-range detection, this paper presents a short-range detection method based on laser polarization properties of targets, designs the polarization detection model and constructs the polarization detection system. Through the analysis and the comparison of data which is obtained from the polarization characteristic and intensity characteristic experiments of five different targets, the conclusion that the detection method based on the laser polarization characteristics can effectively distinguish target is achieved. Meanwhile, the analysis of detection model in this paper can also provide certain theory significance for further research on polarization detection.
\end{abstract}

Keywords- Laser Short-range Detection; Polarization; Detection Model; Polarization Characteristics

\section{INTRODUCTION}

The first problem of short-range detection with laser is to identify the target from the ground objects. False alarm will often appear in target detection, which using only intensity information of retroreflection laser, when the echo intensities of ground objects and target are similar. In addition, the laser short-range detection system can also be interfered effectively by emitting a certain laser beam to the detector from an interference source ${ }^{[1]}$. According to the above limitations of short-range detection system, a new short-range detection method, which is based on polarization characteristics of laser and obtains target information through the polarization of retroreflection laser, can effectively distinguish the target and ground objects, thus obtains higher anti-interference capability in shortrange detection with laser.

When emitting laser beam with certain polarization state to the surface of some object, the polarization state of the reflected or backscattered laser from the object will change, which is related to the its nature, such as material, surface roughness and coatings. Based on this property, a shortrange detection model based on the polarization characteristics of laser is advanced and a short-range detection system with polarized laser is constructed. The analysis on polarization characteristics of different objects is made, and then the detection model and the analytical results are verified by experiments.

\section{PRinciple of Polarization Detection}

\section{A. Description of Polarization State}

Any polarization state of light, including completely polarized light, partly polarized light and unpolarized light, can be described by Stokes vector $\boldsymbol{S}=\left[S_{0} ; S_{1} ; S_{2} ; S_{3}\right]^{\mathrm{T}}$. Because of monochromatic approximation for a plane wave, the four Stokes parameters $S_{0}, S_{1}, S_{2}$ and $S_{3}$ are obtained from the formulas ${ }^{[2]}$ :

$$
\left\{\begin{array}{l}
S_{0}=E_{p} E_{p}^{*}+E_{s} E_{s}^{*} \\
S_{1}=E_{p} E_{p}^{*}-E_{s} E_{s}^{*} \\
S_{2}=E_{p} E_{s}^{*}+E_{s} E_{p}^{*} \\
S_{3}=i\left(E_{p} E_{s}^{*}-E_{s} E_{p}^{*}\right)
\end{array}\right.
$$

where $E_{p}=E_{0 p} \exp \left(i \delta_{p}\right)$ and $E_{s}=E_{0 s} \exp \left(i \delta_{s}\right)$ are complex amplitudes. The subscripts $s$ (i.e. $90^{\circ}$ ) and $p$ (i.e. $0^{\circ}$ ) refer to the two orthogonal components of electric vector of light wave; $E_{0 s}, E_{0 p}$ represent the maximum amplitudes and $\delta_{s}, \delta_{p}$ represent the phases, respectively. The parameter $S_{0}$ represents the total intensity of the light, i.e. the summation of $s$ and $p$ components; the parameter $S_{1}$ represents the intensity difference between $s$ and $p$ components; the parameter $S_{2}$ represents the difference between $+45^{\circ}$ and $45^{\circ}$ polarization; the parameter $S_{3}$ represents the difference between of right and left circular polarization contained within the laser beam.

In order to describe the percentage of polarized light in the total laser beam, DOP (Degree of Polarization) is introduced $^{[2]}$

$$
D O P=\sqrt{S_{1}^{2}+S_{2}^{2}+S_{3}^{2}} / S_{0}
$$


where $\mathrm{DOP}=1, \mathrm{DOP}=0$ and $0<\mathrm{DOP}<1$ represent completely polarized light, unpolarized light and partly polarized light, respectively.

Because the polarization state of laser changes in the transmission, reflection, scattering processes, the Mueller matrix $M$ is introduced in order to describe the transformation from Stokes vector $\boldsymbol{S}_{i}$ of incident light to Stokes vector $\boldsymbol{S}_{o}$ of emergent light, and $\boldsymbol{S}_{o}=M \boldsymbol{S}_{i}$.

\section{B. Method of Polarization Detection}

Figure 1 shows the principle of short-range detection with polarized laser.

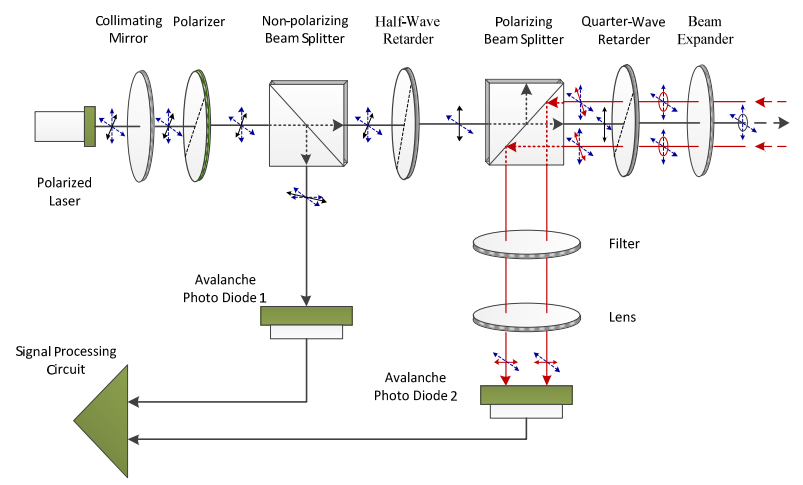

Figure 1. Principle of short-range detection with polarized laser

Completely polarized laser is divided into two parts by non-polarization beam splitter (NPBS), both of the two parts keep their polarization states unchanged, and we choose reflected light as the reference light. After adjusted by halfwave retarder, the polarization direction of transmitted light is the same as transmission direction of polarization beam splitter, i.e. horizontal linear polarized light. Through the quarter-wave retarder with its fast axis at $45^{\circ}$ to the horizontal direction, the transmitted light from the NPBS becomes circularly polarized light. The circularly polarized light irradiate the surface of target, the polarization states of reflected and scattered light change, e.g. reflected light from metal surface becomes elliptically polarized light, and depolarization of the laser beam arises. The retroreflection light becomes partly polarized light through the quarter-wave retarder, and the $p$ component of the partly polarized light is reflected to the receiving optical system after passing by polarization beam splitter (PBS). According to the comparisons between the output voltages of reference light path and receiving optical system, the polarization information can be extracted from the retroreflection light, e.g. we focus on DOP in this paper, and then the different target types can be distinguished.

\section{Equations of Short-Range Detection with Polarized Laser}

Laser detection equation can be regarded as the product of transmission characteristic of optical transmitting system, atmospheric transmission characteristic, target characteristics and receiving system characteristic.

$$
P_{\text {rec }}=P_{\text {opt }} \times \eta_{\text {atm }} \times \rho_{\text {tar }} \times R_{\text {det }}
$$

The first factor $P_{\text {opt }}$ of right part of equation (3) is output power of optical transmitting system.

$$
P_{o p t}=P_{t} G_{t} r
$$

where $P_{t}$ is power of laser, $G_{t}$ is the gain of optical transmitting system, and $r$ is energy distribution ratio of PBS.

The second factor $\eta_{\text {atm }}$ of right part of equation (3) represents the atmospheric transmission characteristic, and atmospheric influence on polarized laser is shown as two main respects: attenuation and depolarization of retroreflection light. The aerosol, which influences the polarization state of laser, can be regarded as a kind of special target, so we only consider atmospheric attenuation of polarized laser. The experience formula of atmospheric transmission characteristics can be expressed as ${ }^{[3]}$

$$
\eta_{a t m}=\frac{3.91}{R_{V}}\left(\frac{\lambda}{550 \mathrm{~nm}}\right)^{-q}
$$

where $R_{V}$ is atmospheric visibility, $\mathrm{km}$; $\lambda$ is wavelength, $\mathrm{nm}$; $q$ is wavelength compensative factor, its value is

$$
q= \begin{cases}1.6 & R_{V}>50 \mathrm{~km} \\ 1.3 & 6 \mathrm{~km}<R_{V}<50 \mathrm{~km} \\ 0.16 R_{V}+0.34 & 1 \mathrm{~km}<R_{V}<6 \mathrm{~km} \\ R_{V}-0.5 & 0.5 \mathrm{~km}<R_{V}<1 \mathrm{~km} \\ 0 & R_{V}<0.5 \mathrm{~km}\end{cases}
$$

When retroreflection light with complicated polarization state incomes, the PBS only reflects the s component of incident light. Hence the second factor $R_{\text {det }}$ of right part of equation (3) relates to not only the gain and aperture of optical receiving system, but also the reflection characteristics of PBS. The factor $R_{\text {det }}$ can be expressed as $R_{d e t}=G_{r} G_{s}$ where $G_{s}$ is the factor that represents the reflection characteristics of PBS. It is not difficult to recognize that $G_{s}$ mainly depends on the polarization state of retroreflection light and has nothing to do with the optical receiving system. Hence $G_{s}$ can be discussed together with target characteristics, and the gain $G_{r}$ is regarded as the only variable of the factor $R_{d e t}$ of receiving system.

Target is regarded as extended target in short-range detection (distance less than $50 \mathrm{~m}$ in this paper), and the laser beam totally irradiates the surface of target. Because can't be assumed for the standard Lambert surface, the laser intensity scattering and reflectance characteristics of rough surface of target is usually described by bidirectional reflectance distribution function (BRDF) ${ }^{[5,6,7]}$.

A five-parameter semi-empirical model is ${ }^{[5]}$ 


$$
\begin{gathered}
f_{r}\left(\theta_{i}, \varphi_{i}, \theta_{r}, \varphi_{r}\right)=k_{b} \frac{k_{r}^{2} \cos \alpha}{1+\left(k_{r}^{2}-1\right) \cos ^{2} \alpha} \exp \left[b(1-\cos \gamma)^{a}\right] \frac{G\left(\theta_{i}, \varphi_{i}, \theta_{r}, \varphi_{r}\right)}{\cos \theta_{r} \cos \theta_{i}}+\frac{k_{d}}{\cos \theta_{i}} \\
\cos \alpha=\frac{\cos \theta_{i}+\cos \theta_{r}}{2 \cos \gamma} \quad \cos ^{2} \gamma=\left(\cos \theta_{i} \cos \theta_{r}+\sin \theta_{i} \sin \theta_{r} \cos \varphi_{r}+1\right) / 2
\end{gathered}
$$

where parameters $k_{b}, k_{d}, k_{r}, a, b$ are unknown and usually obtained by fitting of experimental data. $G$ is a shadowing function and its unknown parameters are usually obtained from experience data. $\theta_{i}, \varphi_{i}, \theta_{r}, \varphi_{r}$ are the zenith angle and azimuth angle of incident light and reflected light respectively. $\alpha$ is the angle between the surface normal and the $\mathrm{Z}$ axis. $\gamma$ is the incident angle of the local coordinates. Because of the transceiver integration design of the detection system, the zenith angle and azimuth angle of incident light and reflected light have the same value.

Based on the BRDF of laser intensity, polarization bidirectional reflectance distribution function (PBRDF) is introduced to short-range detection equation with polarized laser combining with definition of Mueller matrix in section A.

$$
F_{r}\left(\theta_{i}, \varphi_{i}, \theta_{r}, \varphi_{r}\right)=\left(\begin{array}{ccc}
f_{r}^{(0,0)} & \ldots & f_{r}^{(0,3)} \\
\vdots & \ddots & \vdots \\
f_{r}^{(3,0)} & \ldots & f_{r}^{(3,3)}
\end{array}\right)
$$

Here BRDF $f_{r}\left(\theta_{i}, \varphi_{i}, \theta_{r}, \varphi_{r}\right)$ is abbreviated to $f_{r}$.

In actual measurement, the Mueller matrix of surface element is calculated by measuring the Stokes vector of incident and reflected light respectively, and then each function element of PBRDF is fitted according to the Mueller matrix.

Supposing that the Stokes vector of incident circularly polarized light $\boldsymbol{S}_{\boldsymbol{i}}$ is $[1 ; 0 ; 0 ; 1]^{\mathrm{T}}$, the reflection characteristics $G_{s}$ of PBS, which can be regarded as a vertical polarizer when considering the selection characteristic of itself, becomes a Mueller matrix of vertical polarizer $^{[2]}$. Considering equations (7) to (8), the Stokes vector of the light into the optical receiving system can be expressed as

$$
\begin{aligned}
& S_{r}=G_{s} F_{r}(\theta, \varphi) S_{i} \\
& =\frac{1}{2}\left[\begin{array}{cccc}
1 & -1 & 0 & 0 \\
-1 & 1 & 0 & 0 \\
0 & 0 & 0 & 0 \\
0 & 0 & 0 & 0
\end{array}\right]\left[\begin{array}{ccc}
f_{r}^{(0,0)} & \ldots & f_{r}^{(0,3)} \\
\vdots & \ddots & \vdots \\
f_{r}^{(3,0)} & \ldots & f_{r}^{(3,3)}
\end{array}\right]\left[\begin{array}{l}
1 \\
0 \\
0 \\
1
\end{array}\right]
\end{aligned}
$$

Here BRDF $f_{r}\left(\theta_{i}, \varphi_{i}, \theta_{r}, \varphi_{r}\right)$ is also abbreviated to $f_{r}$. Thus the Stokes vector of reflected light after calculation is shown as equation (10). It is evident that the intensity of $p$ component light onto APD2 is $S_{r 0}$ times as much as the output power of optical transmitting system.

$$
S_{r}=\frac{1}{2}\left[\begin{array}{c}
f_{r}^{(0,0)}-f_{r}^{(1,0)}+f_{r}^{(0,3)}-f_{r}^{(1,3)} \\
f_{r}^{(1,0)}-f_{r}^{(0,0)}+f_{r}^{(1,3)}-f_{r}^{(0,3)} \\
0 \\
0
\end{array}\right]
$$

Supposing that the irradiation area of incident light is $S$, the target characteristic factor can be written as

$$
\rho_{\text {tar }}=\frac{1}{2} \int_{S}\left[f_{r}^{(0,0)}-f_{r}^{(1,0)}+f_{r}^{(0,3)}-f_{r}^{(1,3)}\right] d s
$$

Therefore, the key of short-range detection equation with polarized light is the construction of PBRDF model, which is obtained from the comprehensive analysis of equations (3) to (11).

\section{EXPERIMENTS OF TARGET POLARIZATION CHARACTERISTIC}

\section{A. Experiment Platform}

The experiment platform of short-range detection system with polarized laser is shown in figure 2. The Nd:YAG pulsed laser, whose wavelength is $1064 \mathrm{~nm}$ and pumping source is $808 \mathrm{~nm}$ semiconductor laser, provides the polarized light. The splitting ratio of non-polarization beam splitter is $\mathrm{R}: \mathrm{T}=1: 9$.

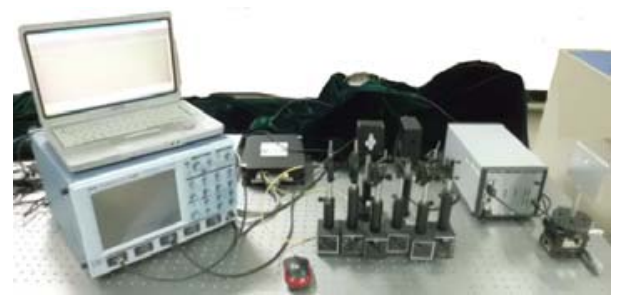

Figure 2. Experiment platform of short-range detection system with polarized laser

\section{B. Analysis of Experimental data}

Using the platform shown in figure 2, several boards of different materials, i.e. steel, aluminum, coated wood, plastic and cement, are set as targets, and then the test for 
degree of polarization of these targets is performed. Figure (3) shows the polarization changes of retroreflection light from different targets.

As shown in (a) of figure 3, when the incident laser irradiates targets at a certain angle, the degree of polarization of retroreflection light varies according to the targets of different materials. When the incident angle is zero, the steel and aluminum have larger DOPs, which are more than 0.9 , followed by plastic whose value is about 0.77 . The DOP of coated wood is about 0.59 , which is greater than plastic, and the cement has the smallest value, which is about 0.3. As the incident angle increases, whether clockwise or counterclockwise, DOPs changes are different with different targets. The DOPs of steel and aluminum decrease as the incident angle increases, become to 0.26 at $\pm 20^{\circ}$, and then change little as the angle increases. The DOP of plastic changes smoothly as the incident angle increases, which becomes to 0.55 rapidly at $\pm 5^{\circ}$, and then decrease to 0.24 as the angle increases. The change of coated wood is similar to the two metals' between 0 and $\pm 20^{\circ}$, but the coated wood has smaller DOP at some certain angle and the DOP comes near to the two irons' as the angle increases between $\pm 20^{\circ}$ and $\pm 40^{\circ}$. The DOP of cement change little as the incident angle increases and comes to 0.23 when the incident angle is $\pm 40^{\circ}$. Moreover, the DOPs of these targets eventually go down to about 0.2 due to the interference of natural light, lamplight and so on in the laboratory environment.

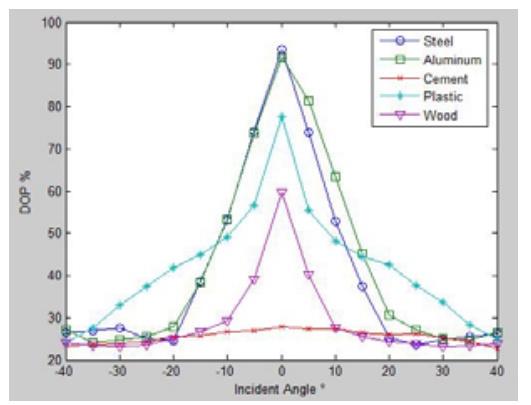

(a)

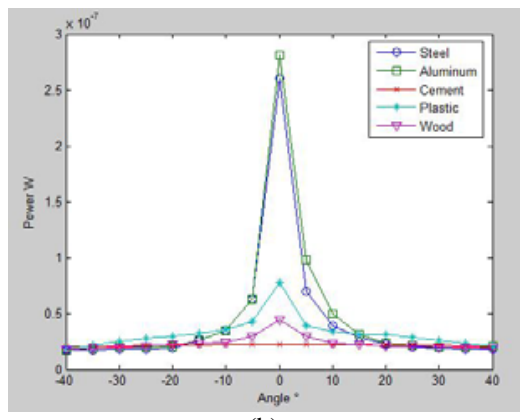

(b)

Figure 3. Changes with different incident angles and materials: (a) DOP and (b) intensities

The (b) of figure 3 shows the intensity change of retroreflection light at different angles under the same experimental conditions of DOP test. When the incident angle is zero, the steel and aluminium reflected the largest intensities, the plastic takes the second place, the coated wood take third place, followed by the cement, and the latter three are obviously different with the metals. The intensities of laser reflected from different targets decrease with different trends as the incident angle increases between $0^{\circ}$ and $\pm 5^{\circ}$, where the metals have the largest rate. There is no outstanding difference between the intensities of retroreflection light from different targets when the incident angle is $\pm 5^{\circ}$, and the intensities converge as the angle increases. Moreover, the intensities are not zero due to the light, lamplight and so on in the laboratory environment.

\section{CONCLUSIONS}

Two conclusions are achieved based on the analysis of experimental data:

- The polarization characteristics have better performance than intensity characteristics in target discrimination. The intensity characteristics are effective in the range between $0^{\circ}$ and $\pm 5^{\circ}$, and the polarization characteristics are applicable in a larger range.

- The DOPs of the retroreflection light from different targets are different at different incident angles, and the change laws are also different obviously. Usually, the metals have larger DOPs due to their smooth surface; the cement has smaller DOP for its rough surface, and the plastic and coated wood lie in the middle. The DOPs of metal and coated wood change acutely as the incident angle increases and the DOPs of plastic and cement vary smoothly.

The principle and platform, which are referred to in this paper, are effective in short-range detection as shown by the analysis of experiments. Meanwhile the detection model, presented in this paper, can provide some theory basis to further study on the polarization properties of targets.

\section{ACKNOWLEDGMENT}

The research was supported by the science and technology foundation of state key lab of mechatronics engineering and control (Grant No. 9140C360707070C11BQ01).

\section{REFERENCE}

[1] Zhiwei Zhou, Xiaoquan Sun, and Xiang Fan, "Jamming methods on laser range finder and relational counter measures,” Infrared and Laser Engineering, vol. 34, no. 6, pp.646-650, 2005.

[2] Dennis Goldstein, Polarized Light, second ed. Marcel Dekker Inc., New York, 2005.

[3] I I Kim, B McArthur, and E Korevaar, “Comparison of laser beam propagation at 785 and $1550 \mathrm{~nm}$ in fog and haze for optical wireless communications," Optical Wireless communications III , vol. 4214, pp.26-37. 2001.

[4] Yongjiang Dai, Lidar Technology, Electronic Industry Press, Beijing, 2010. 
[5] Zhensen Wu, Donghui Xie, Pinhua Xie and Qingnong Wei, "Modelling Reflectance Function from Rough Surface and Algorithms," Acta Optica Sinica, vol. 22, no. 8, pp.898 901, 2002.

[6] Baishun Zhang, Wenqing Liu, Qignong Wei et al., "Experiment measurements and validating with the model of typical goals
BRDF," Chinese Journal of Quantum Electronics, vol. 23, no. 4, pp.533 536, 2006.

[7] L Bousquet, S Lachrade, S Jacquemoud, and I Moya, "Leaf BRDF measurements and model for specular and diffuse components differentiation,” Remote Sensing of Environment, vol. 98, no. 2 , pp. 201 211,2005 